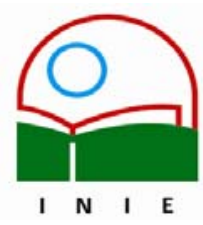

Universidad de Costa Rica

Facultad de Educación

Instituto de Investigación en Educación

ACTUALIDADES INVESTIGATIVAS EN EDUCACION

\title{
HACIA UNA EDUCACIÓN NO SEXISTA
}

\section{Sandra Araya Umaña ${ }^{1}$}

Resumen: El reconocimiento de las expresiones del sexismo comporta un desafío educativo en la medida que la educación debe contribuir a su superación. La tarea es compleja pues la educación es parte de un proyecto global y, por ende, es imposible separarla del contexto social en el que se inserta. Sin embargo, los cambios operados en las últimas décadas en la condición de las mujeres, así como la inclusión de la equidad en la agenda nacional e internacional, obligan a un replanteamiento del devenir de los sistemas educativos. Es en este sentido que las actitudes y las creencias de los y las docentes deben ser revisadas con el fin de que (re) descubran el carácter construido de lo considerado como natural para que así visualicen las desigualdades que encubren estas naturalizaciones. La herramienta analítica que provee esta reconstrucción, es la categoría del género en la medida que constituye una nueva veta teórica en la explicación de la desigualdad de las mujeres.

\section{Palabras clave: GÉNERO/SEXISMO/EDUCACIÓN EMANCIPADORA/}

Abstract: The acknowledgement of the expressions of sexism implies an educative challenge as education must contribute to its improvement. The task is complex because education is part of a global project and, therefore, it is impossible to separate from the social context to which it belongs. Nevertheless, the changes performed during the last decades in the condition of women in general, as well as the addition of gender equity in the national and international agenda, force a new planning of the future development of education systems. Is in this sense that the attitudes and beliefs of the teachers must be revised with the purpose of (re)discovering the constructed disposition of what is considered as natural, so that they may see the inequities that cover these "natural" attitudes. The analytical tool provides this reconstruction is the gender category, as long as it constitutes a new theoretical vein in the explanation of female inequity.

Key words: GENDER/ SEXISM/ EMANCIPATING EDUCATION/

\section{Presentación}

El sexismo es una forma de discriminación que utiliza al sexo como criterio de atribución de capacidades, valoraciones y significados creados en la vida social. Es decir, con base en una construcción social y cultural, la sociedad ordena la realidad en dos cajones que respectivamente se señalan "esto es lo femenino" "esto es lo masculino" y, al igual que otras formas de discriminación, tiende a encorsetar a las personas en parámetros impuestos (Morgade, 2001). En este sentido, podría ser que el término sexista suene exagerado o

\footnotetext{
${ }^{1}$ Doctora en Educación de la Universidad de Costa Rica. Licenciada en Trabajo Social de la Universidad de Costa Rica. Investigadora del Instituto de Investigación en Educación (INIE). Trabajadora Social del Ministerio de Educación Pública. Presidenta de la Asociación Herediana para la Prevención y Atención de la Violencia Doméstica (APREVID). Especialista en educación y género.
}

Correo electrónico: sayarau@racsa.co.cr

Artículo recibido: 27 de octubre, 2004

Aprobado: 29 de noviembre, 2004 
desconcertante debido a las transformaciones que, en los últimos años, ha experimentado la condición de las mujeres. Sin embargo, estas transformaciones han producido una igualdad formal que, aunque necesaria, es insuficiente para la igualdad real o equidad entre las mujeres y los hombres.

La diferenciación sexista afecta a las mujeres y a los hombres. Sin embargo, en el sexismo la diferencia se coloca en una escala jerárquica que termina por sostener el argumento que las mujeres son inferiores. Esta condición de inferioridad se perpetúa a partir de mecanismos sutiles algunos y otros no tanto- que terminan por crear y consolidar representaciones sociales (RS) acerca de las mujeres y de los hombres que, a su vez, actúan para transformar la diferenciación en segregación.

¿Puede la educación contribuir con la erradicación del sexismo? Según mi criterio, sí. Para ello, no obstante, es necesario el reconocimiento de la discriminación, de la desigualdad y de la segregación como problemas educativos, pues nadie cambia aquello que no reconoce como problema.

\section{Lo invisible se hace visible}

Desde la sociología de la educación y, en particular, desde la pedagogía crítica, se empezó a perfilar, hace algunas décadas, una tendencia que evidenció el sistema educativo como un espacio investido por el poder, carente de neutralidad y, por lo tanto, creador y legitimador de identidades sociales jerarquizadas. La necesidad de una educación emancipadora y liberadora comprometida con la superación de las desigualdades sociales, por consiguiente, fue un planteamiento que empezó a cobrar fuerza.

Sin embargo, ni en los sesentas y setentas, la sociología de la educación y la pedagogía crítica hicieron suyo el tema de las mujeres como un foco de las alternativas de liberación. Fue la distinción teórica entre sexo y género, introducida en la década del 70 por los estudios feministas, la que colocó las particularidades de lo femenino en el sistema educativo e inició con ello un nuevo debate acerca del papel que cumplía la educación en la perpetuación y reproducción de las desigualdades entre mujeres y hombres (Subirats, 1999).

En efecto, los estudios de género aportaron una nueva veta explicativa acerca de las desigualdades sociales al puntualizar en aquellas provenientes de la distinción entre lo femenino y lo masculino, pues evidenciaron el carácter cultural de estas desigualdades. 
Asimismo, revelaron que las relaciones entre mujeres y hombres ${ }^{2}$, se distinguen, esencialmente, por estar mediatizadas por relaciones de poder cuyo componente principal es la asimetría.

Con la categoría analítica de género, por tanto, se hizo posible el desmontaje de los determinismos biologistas ${ }^{3}$ que señalaban los comportamientos diferenciados de mujeres y hombres como producto del sexo anatómico. Las consideraciones: "Los hombres son agresivos por las hormonas" "Las mujeres son lloronas por su propia naturaleza", son explicaciones que no encuentran cabida en el análisis de género, debido a que esta categoría develó el carácter construido de las estas afirmaciones y de muchas otras de igual tendencia, creando con ello, las condiciones para una crítica a la existencia de una esencia o naturaleza femenina. Asimismo, posibilitó el reconocimiento de que las mujeres tienen mayores obstáculos para el ejercicio de la autonomía y que los estereotipos de género se han empleado para justificar un trato desigual (Guzmán, 1999).

El género es el conjunto de ideas, representaciones, prácticas y prescripciones sociales que una cultura desarrolla desde la diferencia anatómica entre los sexos, para simbolizar y construir socialmente lo que es propio de los hombres (lo masculino) y lo que es propio de las mujeres (lo femenino) (Lamas, 1999, p. 84).

Existe la tendencia errónea de asociar el género solo con las mujeres y de presumir que hacer estudios con perspectiva de género exige, solamente, la inclusión y distinción de la variable sexo. Como he argumentado en otros escritos (Araya, 2003), si bien para la superación de las desigualdades provenientes del género, es necesario que, en primer término, las mujeres se reconozcan como sujetas de derecho y con condiciones para el desarrollo de un proyecto de vida autónomo, lo cierto es que al referir al mundo de lo social, el género atañe a las relaciones sociales lo cual incluye, relaciones entre hombres y mujeres, entre mujeres $\mathrm{y}$, entre hombres. La perspectiva de género es, entonces, un compromiso teórico y político cuya consecuencia es la superación de las asimetrías entre los sexos (Mosconi, 1998). Implica, por tanto, una acción directa sobre la sociedad para transformar

${ }^{2}$ El género también refiere a la diferenciación sexual, lo que permite dimensionar las desigualdades sociales no sólo entre mujeres y hombres, sino también entre homosexuales y heterosexuales (Lamas, 1996).

3 El biologismo defiende la tesis de que el sexo anatómico determina diferencias en los comportamientos, en las características de personalidad y en las aptitudes de mujeres y de hombres. (Para ampliar, véase Sullerot, Elliot. El hecho femenino: ¿qué es ser mujer?. Barcelona: Argos Vergara. 1979. 
los términos de las relaciones sociales así como una voluntad política para cambiar dichos términos de manera que sea posible la equidad y la igualdad.

Sin desmerecer los cambios ocurridos en las últimas décadas que se expresan, fundamentalmente, en la promoción de leyes y en la paulatina eliminación de los elementos discriminatorios de carácter formal -acceso a la educación, al sufragio electoral y a la propiedad, entre otros- la perspectiva de género, según mi criterio, no ha sido la orientadora de dichos cambios. Prevalecen condiciones societarias que son prueba de ello, a saber:

- La posición de las mujeres en la economía, dado que continúan presentando altas tasas de desempleo y subempleo. De la misma manera y aunque es visible la creciente participación de las mujeres en el mercado de trabajo, se mantiene la brecha salarial en perjuicio de éstas.

- La socialización favorece y estimula alternativas educativas con la subsecuente segregación ocupacional. De esta manera, los hombres dominan en las áreas de ingeniería, informática y seguridad, mientras que las mujeres predominan en la enseñanza y las ciencias sociales que son, por lo general, áreas deficitoriamente remuneradas.

- La subrepresentación en los puestos políticos. A pesar de que en nuestro país y en otros más, se ha establecido la obligatoriedad de nombrar un porcentaje significativo de mujeres en los cargos políticos, la representación femenina no alcanza niveles satisfactorios. Si bien la paridad numérica no es sinónima de paridad genérica, en lo que respecta a la toma de decisiones, ni siquiera se ha alcanzado la primera.

- La violencia intrafamiliar, cuyas principales víctimas son las mujeres, muestra un alarmante aumento, así como un recrudecimiento de sus distintas formas, generando con ello numerosos casos de femicidio. De igual manera, la violencia sexual (incesto, abuso sexual y violación) muestra ritmos crecientes, siendo las mujeres, las niñas y las adolescentes los grupos más afectados.

- Las relaciones de poder y de dominio que caracterizan las relaciones entre mujeres y hombres mantienen intacta su estructura y ello es visible desde diferentes dimensiones:

- La división sexual del trabajo. Si bien han operado cambios en la distribución del trabajo doméstico, existen fuertes evidencias que señalan que los hombres han incorporado con mayor anuencia el cuidado de los niños y las niñas —eso sí en períodos claramente delimitados— que la realización de las distintas tareas (lavar, cocinar, aplanchar, organizar el menú, etc.). Ello es 
revelador de que los hombres (y las mujeres también) siguen considerando el trabajo doméstico inherente a la condición femenina. Es decir, la participación de las mujeres en la esfera productiva no ha tenido un correlato con la participación de los hombres en la esfera reproductiva, generando la doble (y en ocasiones, hasta triple) jornada laboral femenina, la cual, obviamente, también está matizada según la clase social y el nivel ocupacional de las mujeres.

- A pesar de que hay asomo de nuevas formas de masculinidad, continúa prevaleciendo el machismo, es decir, la obsesión del hombre con el predominio y virilidad y que se expresa en posesividad respecto a la propia mujer y en actos de agresión y jactancia con respecto a otros hombres.

- El aumento de los casos de embarazo en niñas y adolescentes y el abandono de los hijos e hijas por parte de los hombres.

- El comportamiento en el manejo y la responsabilidad en las relaciones amatorias, pues las mujeres y los hombres asumen y valoran el amor en forma diferenciada. Mientras que en el caso de los segundos, amar a las mujeres es solamente un elemento —entre otros- de realización posible; en el caso de las primeras, amar a los hombres es un fin en sí mismo (Lagarde, 1994). Esto provoca una tendencia en las mujeres a sentirse garantes de la relación, la cual se acrecienta cuando la relación se fragmenta o finaliza, debido a su presunción de que esto ocurre por su culpa, olvidando la responsabilidad de las dos partes involucradas.

- La educación superior a la que ha tenido acceso un sector de mujeres no se ha traducido en la eliminación de las relaciones desiguales de pareja. En el mejor de los casos, adquiere expresiones más sutiles y, en el peor, se han mantenido invariables.

Mientras prevalezcan expresiones como las anteriores, difícilmente se puede hablar de relaciones igualitarias. Para ello, se precisa una transformación cultural y social que se puede iniciar en la educación.

Sin pretender hacerla depositaria de elementos omnipotentes, la educación puede ser el punto en que se inicie un proyecto emancipatorio de transformación genérica, pues un cambio en algún punto del sistema social repercutirá en todo el sistema en general. Es urgente y en la actualidad se demanda una educación que haga un reconocimiento de la desigualdad procedente de la construcción cultural del género. No se trata de discursos que 
impliquen la aceptación del otro o la otra o de la denominada "cultura de la tolerancia" — el término sugiere todavía una jerarquía en la que, quien tiene poder para ello, acepta convivir con lo distinto en la forma de una concesión o de un acto de magnanimidad - sino de incorporar el análisis de la diversidad y la diferenciación en la educación y en cada uno de sus niveles.

\section{Sexismo y educación}

Es imposible separar la educación del contexto social e histórico en que se inserta. La educación es un fenómeno social y, como tal, tiene una dimensión social y política que complejiza los hechos y las relaciones educativas. Por tanto, el conjunto de relaciones educativas que se entretejen en torno a un hecho educativo, es un microcosmos representativo del cosmos social.

Ello hace que las pautas, los significados, las representaciones sociales y, en general, la ideología sexista, se articulen en las prácticas de las instituciones escolares por medio de diferentes mecanismos: las posiciones que ocupan las mujeres en el sistema de enseñanza; el currículum explícito y el currículum oculto (Apple, 1997; Acker, 1995).

La enseñanza se ha asociado como un trabajo femenino, evidenciando el impacto de la ideología patriarcal que la convierte en una profesión "ideal” para las mujeres y eficaz para preservar la división sexual del trabajo en la esfera doméstica.

...algunos de los argumentos que se utilizaron para abrir la enseñanza a las mujeres recayeron en la reproducción de los elementos que habían formado parte de las causas más arraigadas del control patriarcal: la relación entre enseñanza y domesticidad se estrechó aun más... las mujeres no sólo eran los maestros ideales de los niños pequeños (gracias a su paciencia y sus cualidades para la crianza) sino que la enseñanza era la preparación ideal para la maternidad (Apple, 1997, p. 70).

En diferentes latitudes existe una correlación negativa entre el número de mujeres que ocupan puestos de jerarquía en el ámbito docente y administrativo y el número de mujeres que trabajan en educación. En los niveles de preescolar y de primaria, la gran mayoría de docentes son mujeres; no obstante, la proporción de las mismas disminuye a medida de que aumenta la edad de las alumnas y alumnos y el prestigio social de cada ciclo escolar.

El currículum explícito es el que aparece claramente reflejado en las intenciones que, de una manera directa, indican las normas legales, los contenidos mínimos obligatorios o los 
programas oficiales, los proyectos educativos del centro y el currículum que cada docente desarrolla en el aula (Torres, 1995).

El sexismo en el currículum explícito se refleja en:

- las determinaciones de las políticas acerca de lo que se enseña y no se enseña.

$\square$ el lenguaje que contiene un uso regular y normativo del masculino para designar personas de ambos sexos, incluso cuando la mayoría se compone de mujeres o cuando en el grupo hay únicamente un hombre. Para ello se argumenta el carácter universal del discurso; sin embargo, esta pretendida universalidad oculta que, en realidad, es el sujeto masculino el protagonista central del discurso social.

$\square$ en los textos escolares que por excelencia transmiten papeles tradicionales asignados a hombres y mujeres.

El currículum oculto comprende los aspectos, conocimientos, destrezas, actitudes y valores que se adquieren mediante la participación en procesos de enseñanza y de aprendizaje y, en general, en todas las interacciones que suceden cotidianamente en las aulas y centros de enseñanza. Estas adquisiciones, no obstante, nunca llegan a explicitarse como metas educativas por lograr de una manera intencional (Torres, 1995).

De esta manera, aunque no se explicita, en los ambientes educativos el género es un principio clasificador de las personas (Bonal, 1997) pues, a pesar, de que el profesorado manifiesta que trata por igual al alumnado recurre, a menudo, a las diferencias sexuales para justificar determinados comportamientos. De igual forma, la visión y las expectativas del profesorado, así como las interacciones en el aula, difieren según sea el sexo del alumnado: los hombres se convierten en protagonistas tanto por la cantidad y calidad de atención que reciben, como por el nivel de intervenciones voluntarias que desarrollan (Brullet y Subirats, 1988).

Los textos educativos forman parte del currículum explícito pero, a la vez, del oculto, debido a que las imágenes que aparecen en ellos vehiculizan valores no explicitados que conllevan pautas y tradiciones sexistas (papeles asignados a mujeres y hombres, participación política y comunal diferenciada, etcétera)

Por medio de la interrelación de estos tres mecanismos, el alumnado progresivamente va internalizando las conductas permitidas, las prohibidas, los significados que se le asignan a los acontecimientos, a las verbalizaciones y a los objetos con los que entra en contacto. Esta socialización tiene lugar sobre la base de una construcción y reelaboración activas de significados que realizan tanto el alumnado como el profesorado. Se selecciona información 
que permite construir representaciones que funcionan como mecanismos capaces de determinar el ambiente del aula y como determinantes de la actuación.

Es innegable que, en los últimos años, estos mecanismos han empezado a ser combatidos, en ocasiones, por políticas educativas muy puntuales como, por ejemplo, la modificación de los textos escolares o bien por el trabajo tesonero de algunos y algunas docentes. Sin embargo, es necesario trascender de las respuestas individuales y puntuales, debido a que los intentos aislados provocan desgaste, antes de que aparezcan los frutos. En este sentido es necesario consensuar acerca de un área de atención y así, de esta manera, provocar movilización.

Esta área de atención, según mi criterio, lo constituye el abordaje de las actitudes y las creencias de las y los docentes. La modificación de los libros de texto, los cambios en el lenguaje, la modificación del currículum, entre otras, son medidas necesarias pero no suficientes para la consecución de la equidad genérica. Diversas investigaciones (Araya, 2001) dan cuenta de la necesidad de un profesorado crítico y reflexivo acerca de las prácticas sexistas que imperan en la educación para así poder transformarlas.

Sin embargo, es imperioso reconocer que los y las docentes están atravesados y atravesadas por un repertorio de discursos de género, con diferente poder normativo y prescriptivo que les ofrecen distintas formas de percibirse, percibir a la otra y al otro y actuar desde diversas posiciones genéricas. Las personas están interpeladas, además por discursos sociales de clase, etnia, de subcultura, que pueden articularse de manera más o menos fragmentaria y dinámica con las representaciones de género. En conjunto, estos factores configuran la cosmovisión del profesorado que, sumada al poder que dentro del aula tiene, interviene en la resignificación de las políticas educativas. Es decir, podría ser que las políticas educativas no sean lo suficientemente partidarias de la equidad de género y, sin embargo, según sus características, la práctica educativa podría ser una fuerza que actúe a favor o en contra de la creación de condiciones para la construcción de nuevas relaciones genéricas.

No solo la historia personal del profesorado (es decir su pertenencia a un sexo determinado, a una clase social, a una comunidad) configuran su cosmovisión, también intervienen las características de la trama académica en la que se insertó durante su formación como docente. Es por eso que, los procesos de formación inicial deben superar el déficit que suelen tener en relación con el abordaje de estos temas para que se constituyan en semilleros para la reinterpretación de los contenidos hegemónicos de género. Esto 
redundará no solo en la introducción de cambios en la práctica educativa, sino también —y estoy segura de ello- en la vida personal de las y los docentes.

\section{Acerca de la superación del sexismo}

Para abordar el sexismo en la educación, reitero, es necesario reconocerlo como una forma de discriminación contra las mujeres cuya existencia responde a una construcción social y cultural y, de igual manera, reconocer que la institución escolar contiene elementos sexistas. Empero, esto no debe traducirse en términos maquiavélicos que produzcan una interpretación de la escuela como una institución al servicio de la reproducción mecánica del sexismo. Por el contrario, un elemento clave es la comprensión de la institución escolar como una agencia socializadora compleja y dinámica en la que conviven en tensión diversas representaciones de género (Bonder, 2001).

Por lo anterior, uno de los aspectos centrales para la construcción de una educación no sexista, es la comprensión de que los temas de género provocan una intensa movilidad personal que es explicativa de las distintas reacciones que genera y que integran un extenso abanico con multiplicidad de formas (rechazo, temor, simpatía, aceptación, desconfianza, etcétera).

En efecto, abordar el género significa, entre otros:

- Lo tradicionalmente considerado privado se transforma en público (por ejemplo, la violencia intrafamiliar).

- La visibilización de las relaciones familiares y el ejercicio del poder en el ámbito familiar.

- La discusión y reflexión acerca de las vivencias personales, de las relaciones afectivas, de la sexualidad, de las distintas opciones sexuales y de los derechos reproductivos de las mujeres.

- La construcción de nuevas formas de masculinidad y feminidad atravesadas por el respeto a la diferencia y a la diversidad.

Es frecuente, escuchar "Yo estoy de acuerdo con la igualdad entre mujeres y hombres, pero..." y, por lo general, este pero contiene la carga ideológica de los imperativos sociales y culturales que prescriben comportamientos diferenciados según la pertenencia a un sexo. No se trata de negar la diferencia sexual y el desempeño de las funciones biológicas reproductivas de cada uno de los sexos. Sin embargo, aunque todo apunta a la construcción cultural como elemento preponderante, cuánto de la identidad genérica proviene de la 
experiencia sexual y cuánto es producido por esta construcción, son elementos que con toda claridad saldrán a la luz solo cuando hayan desaparecido las prescripciones sociales. Tampoco se trata de la discusión aun no resuelta que gravita alrededor de los términos iguales o diferentes. Las mujeres y los hombres son diferentes pero esto no significa que sean desiguales en derechos y en el desarrollo de una vida plena. Es preciso, por tanto, que las mujeres y los hombres se (re) descubran en una nueva trama de relaciones que les posibilite, la comprensión de la equidad como la transformación del conjunto de normas sociales y de la desaparición del género, por medio de la fusión de los valores, de las actitudes y de las prácticas históricamente atribuibles a las mujeres y a los hombres. De ninguna manera se trataría de suprimir la diferencia, sino más bien que ésta — sea de sexo, cultura, color, de lengua, opción sexual, religión — deje de ser la base sobre la que se funda y legitima una jerarquía de poder para así pasar al reconocimiento de los rasgos constitutivos de la diferencia como rasgos que presentan un gran valor para la vida de la colectividad, portadores de un principio de renovación o renegociación social que los hace deseables por sí mismos (Subirats, 1998).

Esto significa educar en la diferencia y desde la diferencia para validar las características humanas (ternura, deseo, solidaridad y raciocinio-lógico, entre otras) como expresiones del mismo sustrato, no exclusivas de alguno de los sexos y necesarias para la supervivencia humana, en general.

Lo deseable es la construcción de una nueva forma de vida que permita establecer nuevos equilibrios entre lo público y lo privado, el trabajo productivo y el reproductivo. Pero estos nuevos equilibrios no deben estar basados en un regreso a partir en dos el universo social, y en atribuir una mitad a cada uno de los sexos, sino en que todos los individuos, hombres y mujeres, contribuyan equilibradamente a ambos aspectos de la vida. Es decir, al establecimiento de un nuevo pacto, o un nuevo contrato, como se ha dicho a menudo, entre hombres y mujeres, para una partición del trabajo socialmente necesario que no tenga el carácter de la división sexual del trabajo, sino de la asunción individual de parcelas situadas en ambas esferas (Subirats, 1998, p. 61).

El logro de lo expuesto no es una tarea fácil. Según mi criterio, su consecución requiere de una serie de medidas intermedias entre las que destaca el affidamento ${ }^{4}$ (sin traducción en castellano), es decir, las relaciones de fe, fidelidad y confianza entre las mujeres lo cual

\footnotetext{
${ }^{4}$ Término que proviene de la pedagogía de la diferencia.
} 
incluye las relación entre madres e hijas, entre hermanas, compañeras de trabajo y entre las mujeres, en general.

En lo que respecta al sector educativo, el affidamento permitiría conquistas gremiales y un mayor estatus social y económico, que por su vital función, deben tener la educación y la enseñanza. Asimismo, posibilitaría la superación de las fracturas que por mecanismos patriarcales han producido que las mujeres no se reconozcan como un grupo compacto que, si bien presenta diferencias provenientes de otros ejes (clase social, ciclo de vida, religión y opción sexual, entre otros), comparten las desigualdades sociales provenientes de sus ser femenino.

En este sentido, el significativo peso las docentes en los primeros niveles de la educación, podría convertirse en una de las mayores fortalezas de la emancipación femenina, en tanto contribuyan con una nueva configuración de la educación para que, de acuerdo con Hierro (1998), las mujeres:

- desde niñas, sean orientadas para elegir el tipo de persona que desean ser; el tipo de conocimientos y habilidades que desean adquirir y el tipo de mundo en el que desean vivir.

- formen el carácter infantil no solo para la afectividad, sino para la adquisición y desarrollo de las múltiples capacidades humanas, intelectuales, espirituales y creativas.

- se les prepare para actividades tradicionalmente desarrolladas por los hombres como producir ganancias, administrar negocios y dirigir políticas.

- obtengan una información clara y precisa de su cuerpo y construyan formas renovadas de vivir su sexualidad que trasciendan de su ser "el mal" porque tienen deseos sexuales y de su ser "el bien" porque se mantienen castas.

- alcancen metas de vida que rebasen los intereses centrados en la maternidad.

- revaloren su identidad, más allá de la belleza física y la juventud, para que concedan importancia al ejercicio laboral, la realización personal, la participación política efectiva y la contribución social.

Indiscutiblemente un sector de los hombres comprometido con la superación de las asimetrías será acompañante de estos procesos y hasta podrá tener una participación activa en ellos. Empero, las mujeres tienen el protagonismo y la fuerza que provienen de su condición de subordinación y, la educación, es uno de los mejores mecanismos para potenciarlas. 


\section{Referencias}

Acker, S. (1995). Género y educación. Reflexiones sociológicas sobre mujeres, enseñanza y feminismo. Madrid, España: Narcea.

Apple, M. (1997). Una economía política de las relaciones de clase y de sexo en educación. Barcelona-Buenos Aires- México: Paidós.

Araya, S. (2003). Relaciones sexistas en la educación. Revista Educación. 27 (1): 41-52.

Araya, S. (2001). La equidad de género desde la representación social de los formadores y las formadoras del profesorado de segunda enseñanza. Tesis de Doctorado, Universidad de Costa Rica.

Bonal, X. (1997). Las actitudes del profesorado ante la coeducación. Propuesta de intervención. Barcelona, España: GRAÓ.

Bonder, G. (2001). La equidad de género en las políticas educativas: Lecciones de la experiencia. Ponencia presentada en la Reunión Latinoamericana: Feminismos y Educación. México: Programa Universitario de Estudios de Género.

Brullet, C., Subirats, M. (1988). Rosa y azul. La transmisión de los sexos en la escuela mixta. Madrid, España: Instituto de la Mujer.

Guzmán, L. (1999). Informe de Consultoría. Sistematización y lineamientos para una propuesta teórico-metodológica de trabajo con mujeres adolescentes y jóvenes con perspectiva de género. San José, Costa Rica: Programa Mujeres Adolescentes, Unión Europea ALA 92/46.

Hierro, G. (1998). De la domesticación a la educación de las mexicanas. México: Torres Asociados.

Lagarde, M. (1994). El amor femenino. En Instituto Interamericano de Derechos Humanos. Antología Género y Derechos Humanos. San José, Costa Rica: IIDH.

Lamas, M. (1999). Género, diferencias de sexo y diferencia sexual. Debate Feminista 20. (10) 84-106.

Lamas, M. (comp.). (1996). El género: la construcción cultural de la diferencia sexual. México: Programa Universitario de Estudios del Género.

Morgade, G. (2001). Aprender a ser mujer. Aprender a ser varón. Relaciones de género y educación. Esbozo de un programa de acción. Buenos Aires- México: Novedades Educativas.

Mosconi, Nicole. (1998). Diferencia de sexos y relación con el saber. Buenos Aires, Argentina: Novedades Educativas.

Subirats, M. (1998). La educación de las mujeres: de la marginalidad a la coeducación. Propuestas para una metodología de cambio educativo. Serie Mujer y desarrollo, Santiago, Chile: Naciones Unidas.

Subirats, M. (1994). Conquistar la igualdad: La coeducación hoy. Revista Iberoamericana de Educación (6) 49-75. 
Subirats, M. (1998). Con diferencia: las mujeres frente al reto de la autonomía. Barcelona, España: Icaria.

Subirats, M. (1999). Género y escuela. En Lomas, C (comp.). ¿lguales o diferentes? Género, diferencia sexual, lenguaje y educación. Barcelona, España: Paidós.

Sullerot, E. (1979). El hecho femenino: ¿qué es ser mujer? Barcelona: Argos Vergara.

Torres, J. (1995). El currículo oculto. Madrid, España: Morata

Tovar, T. (1997). Las mujeres están queriendo igualarse. Género en la escuela. Lima, Perú: TAREA Asociación de Publicaciones Educativas. 